\title{
Desenvolvimento de um Modelo da Participação em Bate- papo seguindo a abordagem Design Science Research
}

\author{
Edmilson Barcelos Rocha, Mariano Pimentel, Morganna Carmem Diniz
}

Departamento de Informática Aplicada - Universidade Federal do Estado do Rio de Janeiro (UNIRIO) - Rio de Janeiro - RJ - Brasil

\{edmilson.rocha, pimentel, morganna\} euniriotec.br

\begin{abstract}
This paper describes process and results of a research conduct on the influence of group size on student participation when using educational chat. The research aims to produce a mathematical model to estimate the maximum number of students who must participate in a chat session maintaining the desired level of participation set by the teacher. This research was guided by the method Design Science Research in Information Systems, which conducts performing a behavioral research and the production of an artifact. Communication of this paper aims both technicians in systems modeling and managers in Distance Education (DE).
\end{abstract}

Resumo. Este artigo descreve o processo e os resultados de uma pesquisa realizada sobre a influência do tamanho do grupo na participação em batepapo educacional. A pesquisa tem por objetivo produzir um modelo matemático para estimar a quantidade máxima de alunos que devem participar de um bate-papo mantendo o nível de participação desejado pelo professor. A presente pesquisa foi guiada pelo método Design Science Research em Sistemas de Informação, que orienta a realização de uma pesquisa comportamental e a produção de um artefato. A comunicação do presente artigo visa tanto o público de modelagem de sistemas computacionais quanto gestores que atuam na Educação a Distância (EAD).

\section{Introdução}

A motivação para a realização da pesquisa apresentada no presente artigo é gerar conhecimento sobre o uso do bate-papo na educação. A importância dessa pesquisa decorre do grande crescimento da Educação a Distância no Brasil e o intenso uso de bate-papo nessa modalidade. A partir do ano 2000 ocorreu um grande crescimento da EAD no Brasil: de praticamente zero cursos a distância, em uma década esta modalidade passou a ser responsável por quase $15 \%$ das matrículas na graduação, totalizando cerca de um milhão de estudantes [INEP 2010]. A mídia mais utilizada em cursos a distância ainda é o material impresso: $87,3 \%$ das instituições o utilizam, seguido pelo e-learning $(71,5 \%)$ e pelo vídeo (51,7\%) [CENSOEAD.BR 2010, p.12]. Mesmo o material impresso ainda sendo a mídia mais utilizada, os recursos online têm ganhado cada vez mais espaço por favorecerem um novo paradigma de educação mais voltado para a interação. O bate-papo e o fórum são os meios de interação online mais utilizados na EAD, adotados por mais de $70 \%$ das instituições conforme os dados apresentados no CENSOEAD.BR (2010, p.10).

Na educação online, o uso de sistemas de comunicação síncrono, especialmente o bate-papo, é recomendado por pesquisadores por promover o aumento da participação 
dos alunos [Hrastinski 2007; Stahl 2009]. Mas quantos alunos devem participar de um bate-papo realizado no contexto educacional? Responder a essa pergunta é importante, por exemplo, para planejar a carga horária necessária para realizar uma sessão de batepapo numa turma de um curso a distância. Saber se numa sessão de bate-papo podem participar ao mesmo tempo 100 ou apenas 10 alunos, tem um grande impacto financeiro e no planejamento das atividades de um curso a distância. A informação do limite de participantes em uma sessão de bate-papo educacional é encontrada na literatura de forma imprecisa e conflitante, o que representa um problema relevante, discutido na Seção 3 do presente artigo.

Para saber quantos alunos devem participar de uma sessão de bate-papo educacional, foi construído um modelo matemático da participação de um grupo de alunos durante um bate-papo educacional, apresentado na Seção 4. O modelo concebido, baseado na Teoria das Filas, é um artefato capaz de produzir estimativas objetivadas e corroborar com a pesquisa comportamental sobre a influência do tamanho do grupo na participação dos alunos em bate-papo. O modelo foi desenvolvido por meio de um processo iterativo caracterizado pelo ciclo construção-avaliação, conforme abordado na Seção 5. A avaliação da precisão das estimativas produzidas pelo modelo é apresentada na Seção 6. Na seção 7 são discutidas as contribuições desta pesquisa realizada, que visa tanto o público técnico de modelagem de sistemas computacionais e de comunicação quanto o público gerencial de planejamento de cursos de educação a distância.

A presente pesquisa foi conduzida seguindo as diretrizes de Design Science Research, que é uma abordagem tipicamente adotada na área de Sistemas de Informação, conforme discutido na próxima seção.

\section{Design Science Research}

A pesquisa foi guiada pelas diretrizes do método Design Science Research, proposto na área de Sistemas de Informação [Hevner et al. 2004]. De acordo com Hevner e colaboradores, os conhecimentos necessários para realizar uma pesquisa em SI envolvem dois paradigmas complementares: ciência do comportamento e ciência do design. O paradigma da ciência do comportamento aborda a pesquisa pelo desenvolvimento de teorias que explicam ou predizem fenômenos relacionados à necessidade de negócio identificada. Já o paradigma da ciência de design aborda a pesquisa pelo desenvolvimento e avaliação de artefatos projetados para atender à necessidade de negócio identificada. $\mathrm{Na}$ presente pesquisa, o fenômeno comportamental investigado é a influência do tamanho do grupo na participação em bate-papo educacional; e o artefato desenvolvido é um modelo matemático da participação nessas sessões de bate-papo.

Em Design Science Research, o artefato é projetado com base nas conjecturas teóricas fundamentadas na Ciência do Comportamento; e a avaliação do artefato fornece novos dados sobre as conjecturas elaboradas visando aumentar o conhecimento teórico. $\mathrm{Na}$ Figura 1, é ilustrado o ciclo Design Science Research adaptado à presente pesquisa. 


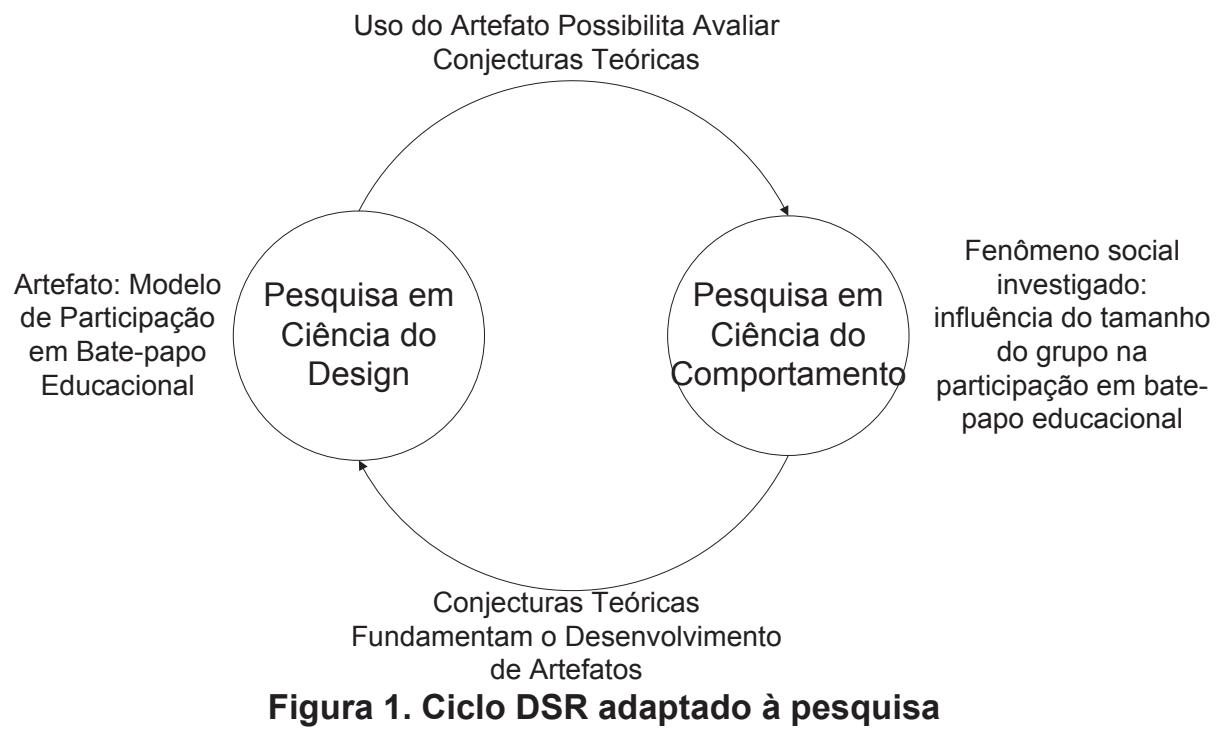

Design Science Research também disponibiliza um conjunto de diretrizes que as pesquisas em Sistema de Informação devem apresentar. Na Tabela 1 estão as diretrizes propostas por Hevner et al. (2004) e a aplicação dessas diretrizes na presente pesquisa.

Tabela 1. Diretrizes do Design Science Research

\begin{tabular}{|c|c|}
\hline DIRETRIZ & APLICAÇÃO DA DIRETRIZ NESSA PESQUISA \\
\hline $\begin{array}{l}\text { Relevância do } \\
\text { Problema } \\
\text { (Seção 3) }\end{array}$ & $\begin{array}{l}\text { Problema: Não se sabe ao certo quantas pessoas devem participar de } \\
\text { uma sessão de bate-papo educacional. } \\
\text { Relevância: A EAD contém } 15 \% \text { das matrículas de graduação no Brasil, } \\
\text { e o sistema de bate-papo é uma das mídias mais adotadas para dar } \\
\text { suporte à tutoria online, sendo adotado por mais de } 70 \% \text { das instituições } \\
\text { que oferece apoio tutorial. }\end{array}$ \\
\hline $\begin{array}{l}\text { Artefato } \\
\text { (Seção 4) }\end{array}$ & $\begin{array}{l}\text { O artefato produzido pela pesquisa é um modelo matemático para } \\
\text { estimar o número de mensagens enviadas em função da quantidade de } \\
\text { participantes na sessão de bate-papo. Esse modelo é a base para } \\
\text { responder a questão da pesquisa, que é determinar a quantidade de } \\
\text { pessoas que devem participar de uma sessão de bate-papo educacional. }\end{array}$ \\
\hline $\begin{array}{l}\text { Processo de } \\
\text { Busca da Solução } \\
\text { (Seção 5) }\end{array}$ & $\begin{array}{l}\text { Método de Design: A construção do artefato foi realizada utilizando a } \\
\text { Modelagem Matemática com Teoria de Filas (KLEINROCK, 1975) } \\
\text { [Seção 5]. O modelo de participação em bate-papo foi desenvolvido num } \\
\text { processo iterativo em que, a cada iteração, o modelo foi alterado com } \\
\text { base nas avaliações realizadas e em novas conjecturas teóricas. }\end{array}$ \\
\hline $\begin{array}{l}\text { Rigor da Pesquisa } \\
\quad \text { (Seções 6) }\end{array}$ & $\begin{array}{l}\text { Método de Avaliação: Pesquisa Documental em que foram comparadas } \\
\text { as medidas obtidas com o modelo e as medidas extraídas de sessões } \\
\text { reais de bate-papo realizadas num curso a distância }\end{array}$ \\
\hline $\begin{array}{l}\text { Avaliação } \\
\text { (Seção 6) }\end{array}$ & $\begin{array}{l}\text { A avaliação do modelo foi baseada na comparação dos resultados } \\
\text { obtidos do modelo e os dados extraídos de } 142 \text { sessões reais de bate- } \\
\text { papo realizadas num curso a distância. Considerando o Teorema Central } \\
\text { do Limite, } 95 \% \text { dos valores reais devem estar contidos a uma distância } \\
\text { de duas vezes o desvio padrão (para mais ou para menos) do valor } \\
\text { estimado pelo modelo para o número de mensagens enviadas pelos } \\
\text { participantes - conforme discutido na Seção } 6 \text {. E de fato esse valor foi } \\
\text { obtido, o que é um indício da adequação do modelo. }\end{array}$ \\
\hline
\end{tabular}




\begin{tabular}{cl}
\hline $\begin{array}{c}\text { Contribuições da } \\
\text { Pesquisa } \\
\text { (Seção 7) }\end{array}$ & $\begin{array}{l}\text { As contribuições da pesquisa são: um modelo da quantidade de } \\
\text { mensagens enviadas em função da quantidade de participantes de uma } \\
\text { sessão de bate-papo educacional; e um maior entendimento sobre a } \\
\text { participação em bate-papo educacional. }\end{array}$ \\
\hline & $\begin{array}{l}\text { A comunicação do artigo visa o público técnico de Modelagem de } \\
\text { Sistemas de Computação e Comunicação (por descrever o processo de } \\
\text { condução da pesquisa) e também o público responsável pela gestão de } \\
\text { cursos de graduação à educação (por disponibilizar um artefato para } \\
\text { Pesquisa } \\
\text { (Seção 7) }\end{array}$ \\
$\begin{array}{l}\text { dimensionar a quantidade de alunos em sessões de bate-papos } \\
\text { educacionais). }\end{array}$
\end{tabular}

O método Design Science Research orienta que a pesquisa deve ser conduzida pela aplicação de métodos rigorosos tanto na construção quanto na avaliação do artefato.

\section{Relevância do Problema}

O objetivo das pesquisas em Sistemas de Informação é produzir conhecimento que possibilite o desenvolvimento e implementação de soluções baseadas em tecnologias para problemas importantes de negócio até agora não resolvidos [Hevner et al. 2004]. Como apresentado na Introdução, o número de alunos que optam por realizar sua graduação na modalidade a distância tem aumentado a cada ano e sistemas de comunicação, como o fórum e o bate-papo, são muito usados na educação online. Muitos pesquisadores apontam as vantagens do uso de sistemas síncronos, como o batepapo, na educação online. Hratinski (2008a) defende que o uso de sistemas síncronos em conjunto com sistemas assíncronos contribuem para o aumento da participação dos alunos na educação online. Silva (2013) afirma que interface de bate-papo é o espaço virtual mais próximo da sala de aula tradicional e, por ser síncrono, permite a construção do sentimento de pertencimento. Apesar da grande popularidade e reconhecida utilidade, é preciso gerar mais conhecimento sobre o uso do bate-papo no contexto educacional. $\mathrm{Na}$ presente pesquisa, buscou-se equacionar quantos alunos podem participar de uma sessão de bate-papo sem decair a qualidade esperada pelo professor.

Stahl (2009) chama atenção para o impacto do tamanho do grupo sobre a eficácia dos diferentes meios de comunicação; ele defende que grupos pequenos, de três ou quatro alunos ativos, são mais complexos e interessantes do que uma dupla respondendo um ao outro. Contudo, se o grupo for grande demais ocorrerão problemas. A "confusão no bate-papo" [Fuks et al. 2006] é definida quando os participantes não conseguem acompanhar a discussão pela ocorrência de fenômenos, como por exemplo, a Sobrecarga de Mensagens causada pela grande quantidade de mensagens postadas em um curto intervalo de tempo decorrente de um número excessivo de participantes na sessão de bate-papo.

Alguns autores, a partir de dados empíricos e em contextos específicos, indicam que o número ideal de participantes num bate-papo é entre 4 e 5 pessoas [Böhlke 2003]; há quem defenda que o bate-papo é mais produtivo com 7 ou mais participantes [Löber et al. 2007]; há quem defenda que o grupo deve ser inferior a 10 participantes [Hrastinski 2008b]; há quem indique que 10 é um tamanho bom para o grupo de participantes [Piva et al. 2011]; e há quem indique que a quantidade de participantes deve estar entre 6 a 18 alunos [Pimentel 2003]. Alguns sistemas de bate-papo limitam o número de participantes a 30 usuários por sessão, como é o caso do Bate-papo UOL; e há outros sistemas que não estabelecem limites. Qual desses números é o correto? 


\section{Artefato: Modelo de Participação em Bate-papo Educacional}

Nas pesquisas em Sistemas de Informação, desenvolve-se um artefato visando resolver um problema relevante. Na presente pesquisa, foi desenvolvido um modelo matemático para estimar quantos alunos devem participar de uma sessão de bate-papo educacional.

Num bate-papo educacional, diferentemente de um bate-papo recreativo, é importante que todos consigam ler todas as mensagens e que também possam enviar mensagens de vez em quando. Se não fosse essa restrição, não haveria um fator limitante para a quantidade de pessoas no bate-papo. Só faz sentido discutir a quantidade máxima de participantes se for exigido um nível mínimo de participação, pois caso a participação possa ser zero (o aluno não precisa ler nem escrever mensagens), tal como ocorre na transmissão de um vídeo em broadcasting, então não há um limite máximo de alunos que devem participar da sessão, e o professor pode dar uma aula por bate-papo para milhares de alunos conectados ao mesmo tempo desde que somente o professor esteja digitando as mensagens e a participação dos alunos tenda a zero. O problema surge quando se dá a chance para o aluno interagir com o professor e com os colegas - e é para isso que serve o bate-papo. No contexto educacional, não é razoável colocar centenas de alunos conversando ao mesmo tempo, por isso deve-se determinar a quantidade máxima de participantes numa sessão de bate-papo para que seja garantida a leitura de todas as mensagens e que seja possível cada um escrever uma determinada quantidade de mensagens. O número total de mensagens produzidas na sessão aumenta proporcionalmente à quantidade de participantes; conforme aumenta a quantidade de mensagens, o participante gasta mais tempo para ler todas as mensagens e terá menos tempo para enviar novas mensagens, e assim sua produção de mensagens decai com o aumento do número de participantes. O objetivo do modelo desenvolvido nessa pesquisa é equacionar a produção de mensagens em função do tamanho do grupo.

O participante escreve uma mensagem e envia para os outros participantes que, por sua vez, irão ler as mensagens recebidas e interagir escrevendo novas mensagens. Quando o participante está lendo ou escrevendo alguma mensagem e nesse momento chegam outras mensagens a serem lidas, essas são enfileiradas para a posterior leitura. Desta forma, para elaborar o modelo foi empregada a Teoria de Filas [Kleinrock 1975] Figura 2 - em que cada participante é representado como um servidor que precisa ler (servir) todas as mensagens (clientes) digitadas pelos outros participantes, que vão sendo enfileiradas para a leitura na ordem em que são recebidas. Cada participante, além de ser um servidor, também desempenha o papel de uma fonte geradora de mensagens que são enviadas para as filas dos demais participantes.

FILA SERVIDOR

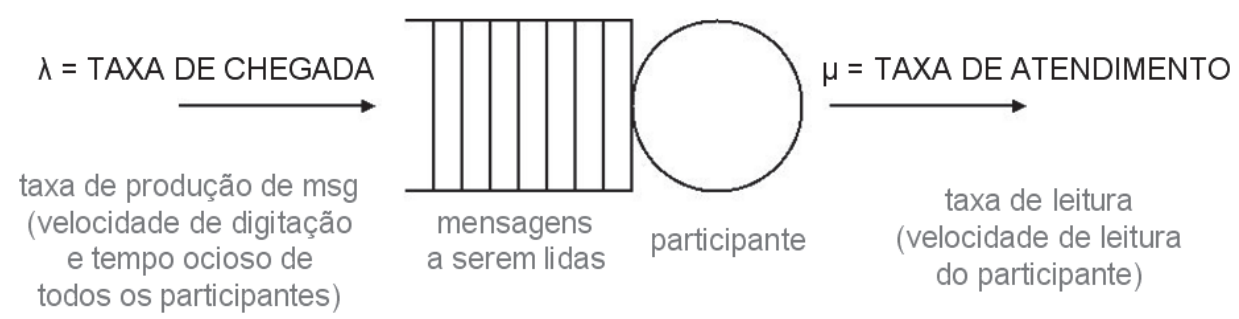

Figura 2. Sistema com fila única

Dentre os parâmetros desse modelo, identifica-se a taxa de leitura de mensagens $\mu$, que é o inverso do tempo médio de leitura das mensagens produzidas na sessão; e a 
taxa de produção de mensagens $\lambda$, que se deseja determinar dado o tamanho $p$ do grupo de participantes. Os tempos de leitura e de produção de mensagens são considerados, na presente pesquisa, como exponencialmente distribuídos.

Para obter as estimativas de produção de mensagem a partir do modelo construído, o modelo foi implementado no Tangram-II [De Souza e Silva et al. 2009]. O Tangram-II é um ambiente de modelagem baseado no paradigma orientado a objetos que fornece uma variedade de solvers para obtenção das medidas de interesse. As estimativas produzidas a partir do modelo elaborado na presente pesquisa foram obtidas a partir da Solução Analítica do Tangram-II.

\section{Processo de Busca de Solução}

O ciclo de design (construção/avaliação) é o coração de todo projeto de pesquisa em ciência de design e também onde é feito o trabalho mais intenso em Design Science Research [Hevner 2007]. Deve-se descrever todo o processo de condução da pesquisa e deve-se aplicar métodos rigorosos tanto na construção quanto na avaliação do artefato. O rigor da pesquisa é derivado do uso eficaz da base de conhecimento, que é o conjunto de fundamentos e metodologias necessárias para a realização da pesquisa.

A cada construção do artefato, os dados produzidos pelo modelo foram comparados com os dados extraídos de um cenário real de bate-papo na educação. Em seguida o artefato foi refinado visando obter estimativas mais precisas.

Para avaliar se o modelo produz estimativas com uma precisão dentro de um limite de aceitação, essas estimativas foram comparadas com dados extraídos de logs reais de bate-papo de turmas de graduação em Informática. Dos logs de bate-papo, foram extraídas a duração do bate-papo, a quantidade de participantes e a quantidade de mensagens enviadas. Espera-se que o modelo, com os parâmetros da turma, produza estimativas próximas dos dados reais.

\subsection{Modelagem do comportamento de um usuário de bate-papo}

Para modelar o comportamento de um participante da sessão de bate-papo educacional, foi desenvolvido um diagrama de estados para representar as atividades primárias que o participante realiza durante o bate-papo: ler e escrever mensagens - Figura 3. Nessa abordagem, o participante permanece no estado Escrevendo por um determinado tempo médio até que envia a mensagem aos demais participantes. Após enviar a mensagem, ele verifica se existem mensagens a serem lidas, e se houver passa para o estado Lendo. Enquanto o participante está Escrevendo ou Lendo mensagens, outras mensagens podem chegar e essas são enfileiradas na ordem em que são enviadas pelos demais participantes em um Fila de Mensagens. A transição de estados é modelada em função de taxas. $\mathrm{O}$ participante permanece Lendo uma determinada mensagem por um tempo médio de leitura $\left(\bar{T}_{L}\right)$, e a taxaLeitura é calculada como o inverso do tempo médio. Da mesma forma, o participante permanece no estado Escrevendo durante um tempo médio de escrita $\left(\bar{T}_{E}\right)$, a partir do qual se calcula a taxa de escrita.

Representar um participante de bate-papo por apenas dois estados é irreal, pois pressupõe que o participante fica o tempo todo ou Lendo ou Escrevendo mensagens sem nunca ficar ocioso. A primeira fase de avaliação mostrou que as estimativas do modelo foram muito superiores comparadas aos dados reais, contudo essa simplificação da realidade é o que possibilita estimar um Limite Máximo de mensagens produzidas pelo 
participante numa sessão de bate-papo, pois pressupõe que esse sujeito se encontra o tempo todo ocupado, produzindo o máximo de mensagens que seria capaz.

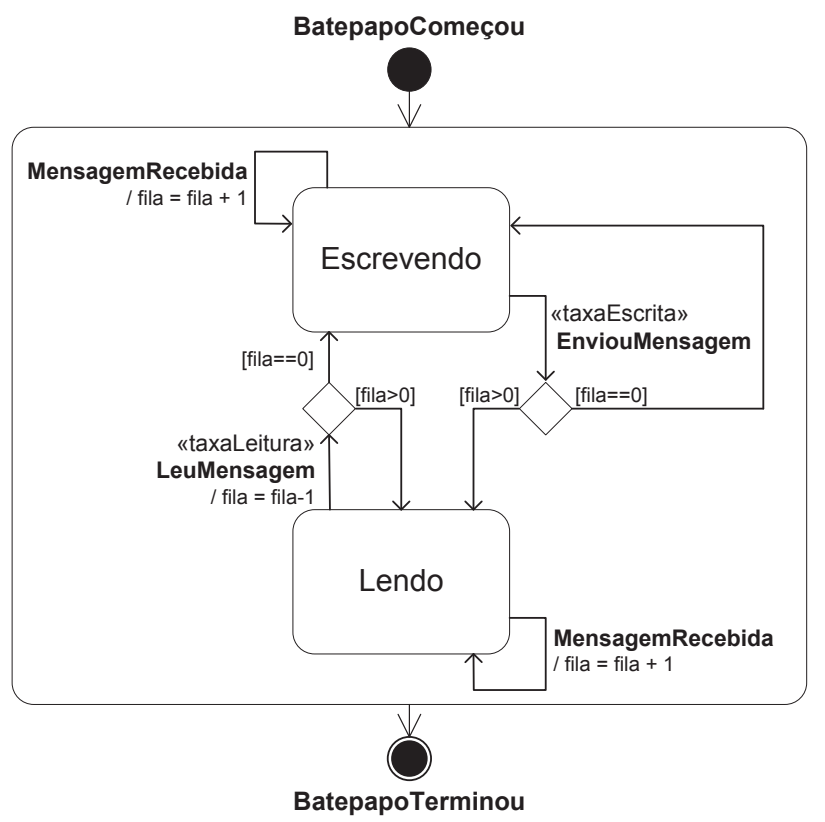

Figura 3. Diagrama dos Estados Lendo e Escrevendo

Uma nova abordagem foi desenvolvida para reduzir a diferença entre o real e o estimado pelo modelo. No segundo diagrama de estados, apresentado na Figura 4, levase em conta que um participante, além de ler e escrever mensagens, também fica refletindo em alguns momentos.

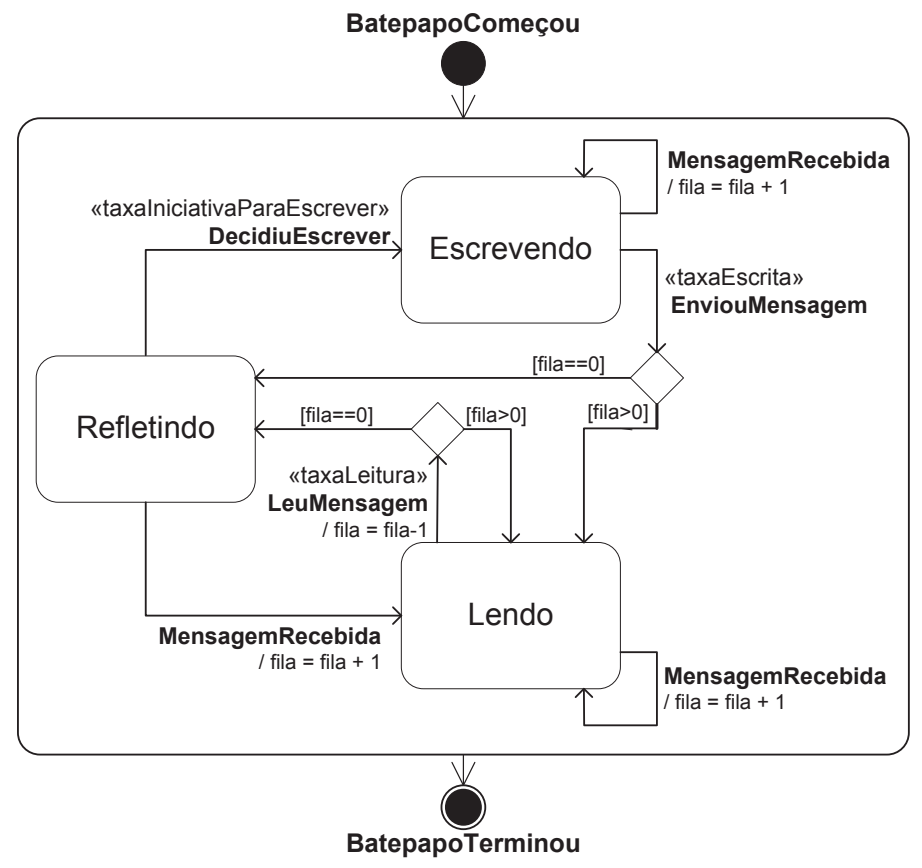

Figura 4. Diagrama dos Estados Lendo, Escrevendo e Refletindo 
Nessa abordagem, o indivíduo vai para o estado Refletindo quando não tem mais mensagens a serem lidas, e fica refletindo por um tempo até que: chega uma nova mensagem a ser lida e, imediatamente, o usuário volta para o estado Lendo; ou fica refletindo por algum tempo até que decide escrever uma nova mensagem, indo para o estado Escrevendo.

O modelo com estes três estados produz estimativas compatíveis com os dados reais utilizados para validação do modelo (apresentado na Seção 6). Contudo, nesta pesquisa chegou-se a desenvolver um modelo ainda mais refinado contendo quatro estados, como documentado por Rocha (2013). Porém, o modelo com quatro estados somente produz estimativas diferentes do modelo aqui apresentado quando há poucos participantes (até 5 ou 6 participantes), e as sessões reais de bate-papo usadas como referência para a comparação não contém tão poucos participantes, e assim não foi possível investigar qual dos dois modelos produz estimativas mais acuradas. Sendo assim, adotamos o diagrama apresentado na Figura 4 por ser mais simples. Esse processo iterativo em busca da construção do artefato que resolva o problema investigado é característico de Design Science Research.

\subsection{Modelagem da interação entre os participantes da sessão de bate-papo}

Conforme apresentado na Seção 4, para se obter as estimativas de produção de mensagens, o modelo foi implementado no ambiente de modelagem Tangram-II, que é baseada no paradigma orientado a objetos. O Modelo de Participação é formado por um grupo de objetos do tipo "Participante" que possui o comportamento descrito no Diagrama de Estados da Figura 4. O objeto Participante é então replicado $p$-vezes até se representar todos os participantes do grupo. A essa estratégia de modelagem foi dado o nome Interação entre Indivíduos, conceitualmente representada na Figura 5.

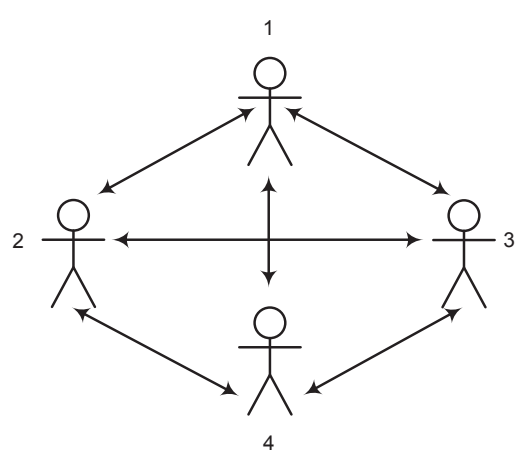

Figura 5. Estratégia de Modelagem: Interação entre Indivíduos

Após o modelo estar implementado no Tangram II (módulo Model Specification), gera-se a Cadeia de Markov contendo o conjunto de todos os estados dos $p$ participantes bem como as transições possíveis entre os estados (módulo Mathematical Model). Segundo Fogliatti e Mattos (2007) "Cadeia de Markov é um processo estocástico com espaço de estados discretos, onde a cadeia é formada por um conjunto de variáveis e a probabilidade do próximo estado depende apenas do estado atual-memoryless". Por fim, as estimativas de produção de mensagens por participante são obtidas pela técnica de análise transiente Expected Cumulative Impulse Reward (módulo Analytical Model Solution). A cada envio de mensagem pelo participante, uma recompensa é adicionada. 
Por meio da estratégia de modelagem Interação entre Indivíduos, só é possível obter as estimativas de mensagens para até 5 participantes, pois com 6 ou mais participantes ocorre uma explosão de estados já que o número de estados da Cadeia de Markov aumenta exponencialmente a medida que o número de participantes é incrementado. Como um dos objetivos da pesquisa é estimar o máximo de participantes que devem participar de uma sessão de bate-papo mantendo uma determinada produção de mensagens, essa solução se mostrou inviável.

Para contornar o problema de explosão de estados do ambiente Tangram-II, foi elaborada uma segunda estratégia de modelagem com apenas 2 objetos: um Participante, com o comportamento descrito no diagrama de estados da Figura 4, e um Grupo de Participantes modelado como uma fonte geradora de mensagens, que produz mensagens emitidas para o Participante a uma taxaEnvioMsg - Figura 6.

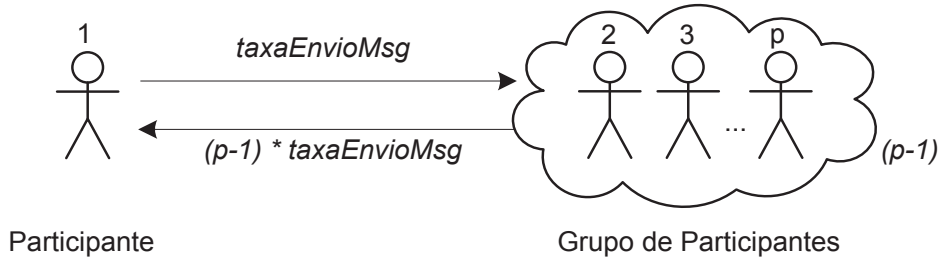

Figura 6. Estratégia de Modelagem: Interação Indivíduo-Grupo

Nessa estratégia, cada participante do Grupo produz mensagens numa taxa semelhante ao do indivíduo isolado, pois assume-se que todos os participantes do batepapo tem comportamento similar. Isto significa que a taxa de envio do Grupo dividida por $(p-1)$ precisa ser próxima ao número de mensagens enviadas pelo indivíduo isolado. $\mathrm{O}$ valor da taxa de mensagens enviadas pelo indivíduo isolado é a medida de interesse que se quer encontrar. Para obter essa taxa, o primeiro passo é atribuir um valor aleatório para a taxa de produção de mensagens pelo grupo e, pelo Tangram-II, calcular a taxa de envio de mensagens do indivíduo isolado. Em seguida compara-se a taxa atribuída ao grupo e a produzida pelo indivíduo. A diferença entre as taxas deve ser a menor possível - na presente pesquisa, foi definida que a diferença deveria ser inferior a 1 mensagem. Interativamente vai se ajustando a estimativa da taxa de envio de mensagens do grupo até que a taxa dos indivíduos do grupo fique próxima da taxa do indivíduo isolado. Desta forma foi possível obter, com o Tangram II, as estimativas para a medida de interesse da presente pesquisa para qualquer tamanho de grupo.

\section{Rigor da Pesquisa: Avaliação do Modelo}

A avaliação do artefato produzido pela pesquisa foi feita pela comparação das estimativas calculadas pelo modelo com os dados reais obtidos de logs de bate-papo das turmas de uma disciplina lecionada totalmente a distância, denominada "Tecnologias de Informação Aplicadas à Educação" (TIAE) [Lucena e Fuks 2002]. Ao todo foram obtidos 142 logs de bate-papo ocorridos em 18 turmas ministradas entre os anos de 1999 e 2008 (em cada turma foram realizadas, em média, 8 sessões de bate-papo). Para realizar a avaliação do modelo, foram extraídas dos $\log s$ de bate-papo das turmas TIAE as seguintes métricas de interesse: duração da sessão de bate-papo, quantidade de participantes, quantidade de mensagens enviadas por participante e quantidade média de caracteres por mensagem.

A parametrização do modelo de participação foi feita com base em estudos 
realizados em uma turma de pós-graduação em informática sobre o processo de leitura e o processo de escrita de mensagens em bate-papo educacional [Rocha 2013]. Foi identificada uma alta correlação entre o tamanho médio da mensagem e os tempos médios de leitura e tempo médio de escrita. Os estudos possibilitaram extrair equações de tempo de leitura e tempo de escrita em função da quantidade de caracteres da mensagem. A quantidade média de caracteres por mensagem extraída dos logs reais serviu de base para parametrizar o modelo. Com a informação da duração média de cada turma e da produção média de mensagens por aluno, foi possível inferir o tempo médio de reflexão dos alunos. Como o modelo todo parametrizado, as estimativas de produção de mensagens para cada tamanho de grupo puderam ser obtidas pelo Tangram-II. Com as estimativas produzidas pelo modelo foi traçada a curva de participação prevista para cada turma TIAE, conforme exemplificado na Figura 7.

TIAE 2007.1

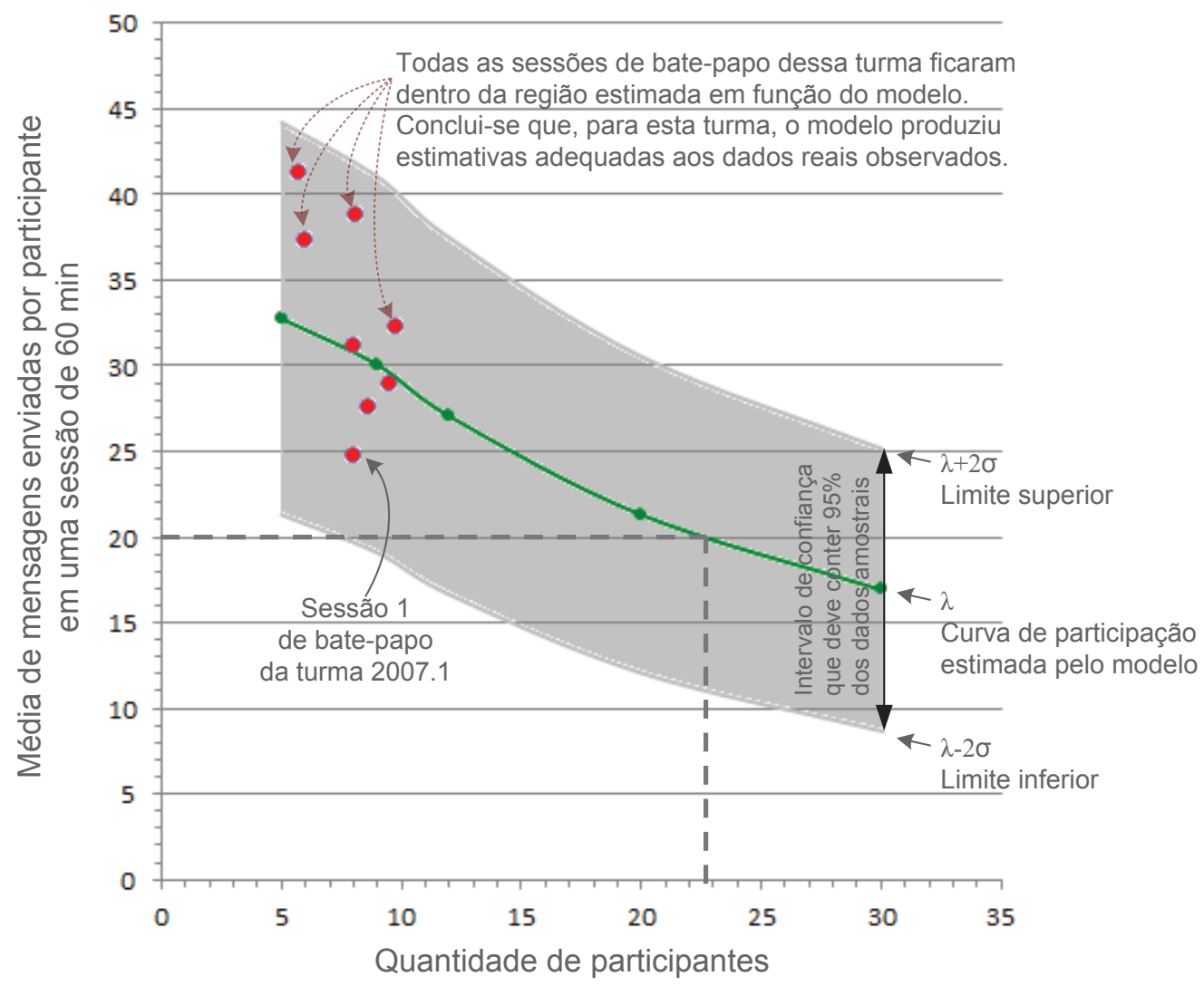

Figura 7. Curva de Participação e Limites Superior e Inferior para Avaliação

A curva de participação é traçada pela união dos pontos estimados pelo modelo de produção de mensagens para cada tamanho do grupo de participantes. Como ilustrado na Figura 7, foi verificado se a quantidade de mensagens produzidas por participante em cada sessão da turma TIAE ficou dentro de certos limites ao redor do ponto estimado pelo modelo. De todas as sessões de bate-papo das turmas TIAE, $95,8 \%$ ficaram dentro da região estimada em função do modelo. Este valor é bem próximo ao valor esperado pelo Teorema Central do Limite $(95,4 \%)$, o que possibilita concluir que o modelo de participação produz estimativas adequadas da média de mensagens enviadas por sessão de bate-papo em função do tamanho do grupo. 
A partir desse modelo é possível responder a questão da pesquisa. A quantidade de alunos que devem participar de uma sessão de bate-papo depende das características da turma (velocidade média de digitação, leitura e reflexão) e da quantidade de mensagens esperadas de cada participante. Por exemplo, considerando-se o contexto da turma da Figura 7, caso fosse esperado que cada participante produzisse ao menos 20 mensagens ao longo da sessão de 60 minutos de bate-papo, identifica-se então que não devem participar mais que 22 sujeitos. Se o professor espera que cada aluno produza 30 mensagens na sessão (1 mensagem a cada 2 minutos), precisa então organizar a turma em grupos ainda menores, e pelo modelo identifica-se que o grupo deveria ter 9 ou menos participantes considerando o perfil médio dos sujeitos daquela turma.

\section{Conclusão: Contribuições da Pesquisa}

$\mathrm{Na}$ presente pesquisa, foi elaborado um modelo matemático para representar o comportamento de usuários em sistemas de bate-papo no contexto educacional, visando determinar qual o número máximo de alunos que devem participar de uma sessão de bate-papo. Os resultados produzidos pelo modelo elaborado mostraram-se adequados em comparação com os dados reais obtidos de 142 sessões de bate-papo realizadas em 18 turmas de uma disciplina a distância, e assim conclui-se que o modelo elaborado nessa pesquisa produz estimativas adequadas. $\mathrm{O}$ modelo é útil para subsidiar diferentes estudos, e nessa pesquisa mostrou-se a utilidade do modelo para apoiar o planejamento do tamanho do grupo de alunos que devem participar de uma sessão de bate-papo educacional - esse resultado é de interesse para as instituições que promovem educação online.

A presente pesquisa também contribuiu para um maior entendimento sobre a teorização da participação em bate-papo educacional. Buscou-se caracterizar o comportamento de participação em função de processos elementares: leitura e escrita de mensagens, e de reflexão. Foi possível equacionar o limite máximo de produção de mensagens em função da quantidade de participantes e, a partir do modelo elaborado, foi possível estimar a quantidade esperada dessa produção de mensagens num dado contexto. Foi possível também verificar a influência do aumento do tamanho do grupo na participação dos alunos. Quanto maior a quantidade de participantes, menor é a produção de mensagens, uma vez que o participante necessita de mais tempo para ler as mensagens dos outros participantes.

\section{Referências}

Böhlke, O. (2003). A comparison of student participation levels by group size and language stages during chatroom and face-to-face discussions in German. CALICO Journal, 21 (1), 67-87.

CensoEAD.BR (2010) Relatório analítico da aprendizagem a distância no Brasil. Associação Brasileira de Educação a Distância. São Paulo: Pearson Education do Brasil <http://www.abed.org.br/censoEAD/CensoEADbr0809_portugues.pdf $>$. Acessado em 18 dez 2012.

De Souza e Silva, E., Figueiredo, D., Leão, R. (2009). The TangramII integrated modeling environment for computer systems and networks. SIGMETRICS Perform.

Fogliatti, M. C. e N. M. C. Mattos (2007) Teoria de Filas. Interciência, Rio de Janeiro.

Fuks, H., Pimentel, M., Lucena, C.J.P. (2006) R-U-Typing-2-Me? Evolving a chat tool 
to increase understanding in learning activities. International Journal of ComputerSupported Collaborative Learning, v. 1, p. 117-142.

Hevner, A., March, S., Park, J., and Ram, S. (2004) "Design Science in Information Systems Research," MIS Quarterly (28:1), 2004, pp. 75-105.

Hevner, A. R. (2007). A Three Cycle View of Design Science Research. Scandinavian Journal of Information Systems, v.19, n. 2, p. 87-92, 2007.

Hrastinski, S. (2007). Participating in synchronous online education. (Doctoral dissertation, Lund University).

Hrastinski, S. (2008a). The potential of synchronous communication to enhance participation in online discussions: A case study of two e-learning courses. Information \& Management, 45, 499-506.

Hrastinski, S. (2008b). What is online learner participation? A literature review. Computers \& Education.

INEP (2010) Censo da Educação Superior 2010. MEC, 2010. Disponível em $<$ http://portal.mec.gov.br/index.php?option $=$ com_docman\&task $=$ doc_download\&gid $=9332 \&$ Itemid $=>$. Acessado em 18 ago 2012 .

Kleinrock, L., (1975), Queueing Systems - Theory, Vol. I. Wiley-Interscience.

Löber, A, Schwabe, G, Grimm, S (2007). Audio vs. chat: The effects of group size on media choice. Proceedings of the 40th HICCS Hawaii International Conference on System Sciences.

Lucena, C.J.P., Fuks, H. (2002). Tecnologias de Informação Aplicadas à Educação (TIAE): Manual do Aprendiz. Monografias em Ciência da Computação n.07/02, PUC-Rio, 2002.

Pimentel, M. G., Fuks, H. e Lucena, C. J. P. (2003) "Debati, debati... aprendi? Investigações sobre o papel educacional das ferramentas de bate-papo", IX Workshop sobre Informática na Escola - WIE2003, Agosto.

Piva, D.J., Pupo, R. Gamez, L., Oliveira, S. (2011). EAD na Prática - Planejamento, métodos e ambientes de educação online. Elsevier Editora Ltda.,2011, v.,p 164-170.

Rocha, E. B. (2013). Modelo de Participação em Bate-papo Educacional. 2013. 123 f. Dissertação (Mestrado em Informática) - Universidade Federal do Estado do Rio de Janeiro.

Silva, M. Avaliação da Aprendizagem em Educação Online. 2010. Entrevista publicada no YouTube: < http://youtu.be/S7uUd6afEYE>. Acessado em 12 ago 2013.

Stahl, G. Studying Virtual Math Teams. New York, NY: Springer, 2009. 